\title{
EMPATÍA Y PENSAMIENTO MÁGICO EN LA FORMACIÓN DE LA ALIANZA TERAPÉUTICA EN PSICÓLOGOS Y TAROTISTAS
}

\section{EMPATHY AND MAGICAL THINKING IN THE FORMATION OF THE THERAPEUTIC ALLIANCE IN PSYCHOLOGISTS AND TAROTTISTS}

\author{
Alejandro Parra \\ Universidad Abierta Interamericana, Argentina \\ Claudia Sciacca \\ Psicóloga, Ciudad de Florencio Varela, Argentina
}

Cómo referenciar este artículo/How to reference this article:

Parra, A. (2017). Empatía y pensamiento mágico en la formación de la alianza terapéutica en psicólogos y tarotistas. Revista de Psicoterapia, 28(106), 149-164.

\section{Resumen}

El objetivo de este estudio es comparar el grado de vinculo que establecen dos estilos de intervención terapéutica, el grado de de empatía (cognitivayafectiva) con el cliente y el pensamiento mágico (creencias paranormales) entre psicólogos clínicos y practicantes de tarot. Aunque ambos grupos difieren en cuanto a objetivos, instrumentos y procedimientos, sin embargo, hay evidencia empírica que sugiere los tarotistas producen cambios actitudinales, cognitivos, emocionales y existenciales significativos - a menudo positivos- en la vida de sus clientes/consultantes. Se administró el Test de Empatía Cognitiva y Afectiva, el Inventario de Alianza Terapéutica, y el Cuestionario de Creencias Paranormales junto a una encuesta de estilos y prácticas de consejería (adaptada apropiadamente) a una muestra practicantes de tarot y psicólogos de orientación clínica. Los resultados sugieren que las emociones y las creencias de los clientes/consultantes no deberían ser un obstáculo sino un "atajo" para empatizar o crear un puente funcional para una psicoterapia exitosa. Aunque no se encontraron diferencias en la construcción de la alianza terapéutica, los psicólogos no priorizaron la empatía con su cliente. De modo que la producción de mayor vínculo cognitivo/ emocional entretarotista/consultante posiblementesea más flexible que el establecido por el psicoterapeutal cliente.

Palabras clave: Empatía, Pensamiento Mágico, Alianza Terapéutica, Psicólogos, Tarotistas.

\begin{abstract}
The aim of this study is to compare the degree of link to establish two types of therapeutic intervention, degree of empathy (cognitive and emotional) with the patient and magical thinking (beliefs framework) among clinical psychologists and tarot readers. Although both groups differ in terms of objectives, instruments and procedures, however, no empirical evidence suggesting the tarot readings produce attitudinal, cognitive, emotional and existential changes, often positives, in their lives. Cognitive and affective empathy, Therapeutic Alliance Inventory, and Paranormal Beliefs Scale with a survey of counseling styles and practices were given to a sample tarot readers and clinical psychologists. The results suggest that emotions and beliefs of clients/ consultants should not be an obstacle but a "bypass" to create a functional bridge to successful psychotherapy. Although no differences in the construction of the therapeutic alliance was found, however psychologists did not prioritize empathy with the clients. Higher cognitivelemotional empathy between tarotistconsultants may be more flexible than that established by the psychotherapist-clients.
\end{abstract}

Keywords: Empathy, Magical Thinking, Therapeutic Alliance, Psychologists, Tarot readers.

\section{Fecha de recepción: 3 octubre 2016. Fecha de aceptación: 24 febrero 2017.}

Correspondencia sobre este artículo:

E-mail: rapp@fibertel.com.ar

(C) 2017 Revista de Psicoterapia 


\section{INTRODUCCION}

La alianza terapéutica se define como un encuentro entre dos o más personas, que acuerdan mantenerse en interacción por un período determinado, para provocar cambios en la organización de uno o más de los participantes en esta interacción (Botella y Feixas, 1998). Lossa, Corbella y Botella (2012) exponen sus ideas sobre la necesidad del establecimiento de la alianza terapéutica considerándola como un elemento esencial en algunos casos, o menos determinante en otros, para el éxito terapéutico; entre ellos, por ejemplo, Ojeda (2010) sostiene que la alianza desempeña un papel fundamental en la relación terapéutica, sugiere que la teoría del apego permitiría ampliar aún más la comprensión de esta relación. Bowlby (1995) coincide con Ojeda en la idea del apego en la alianza, sosteniendo que ésta es cualquier forma de conducta que tiene como resultado el logro o la conservación de la proximidad con otro individuo al que se considera más capacitado para enfrentarse al mundo. Continuando en esta línea, Feeney y Noller (2001) concluyen que algunas relaciones adultas son relaciones de apego.

Numerosas investigaciones advierten la importancia de la alianza terapéutica y su influencia en los resultados positivos de la psicoterapia (Andrade González, 2005; Baringoltz, 2005; Corbella y Botella, 2003). Etchevers, González y Simkin (2012) sostienen la importancia del establecimiento de esta alianza para un resultado favorable en todo tratamiento, dependiendo de la perspectiva psicoanalítica o cognitivo-conductual, también examinan la literatura y señalan la actual concepción que se tiene de la alianza terapéutica.

Corbella y Botella (2003) afirman que no se puede entender la psicoterapia sin la concepción de la alianza terapéutica como una relación interpersonal entre el terapeuta y el cliente. Reconocen la importancia de establecer una relación empática entre cliente y terapeuta para optimizar el tratamiento. Este interés despertó la necesidad de construir instrumentos que permitieran evaluar la alianza terapéutica; en la actualidad hay más de 20 medidas diferentes de alianzas. Ambos autores concluyen que existe una relación significativa entre alianza terapéutica y el resultado final de la psicoterapia. Las conclusiones del trabajo permitieron afirmar la importancia del constructo alianza terapéutica en todo el proceso terapéutico y en los resultados finales.

Sin embargo, Luborsky (1976) definió la alianza terapéutica desde una mirada psicodinámica, sosteniendo que: “(...) es una entidad dinámica que evoluciona con los cambios de las demandas de las diferentes fases de la terapia" (p. 208). Luborsky refiere a dos etapas o momentos de la alianza terapéutica, la primera, que se establece en el comienzo de la terapia y que está relacionada con la experiencia del cliente en relación al terapeuta en quien ve una persona que lo contiene y ayuda; y una segunda etapa posterior al proceso terapéutico y que se caracteriza por la sensación de haber trabajado conjuntamente para alcanzar los objetivos de la terapia. De lo expuesto, puede interpretarse a la alianza terapéutica como el resultado de una construcción conjunta entre el cliente y el terapeuta donde, 
siguiendo a Luborsky, “(...) las expectativas, las opiniones, las construcciones que ambos van desarrollando respecto al trabajo que van realizando, la relación establecida y la visión del otro resultan relevantes para el establecimiento de la alianza terapéutica (...)” (p. 208).

Si bien se han realizado muchos estudios e investigaciones sobre empatía, hay pocos estudios acerca de la empatía con clientes (ver Fernández Pinto, López-Pérez, Márquez, 2008; Olivera, Braun y Roussos, 2011; Piedrabuena, 2007). A finales de los años sesenta, muchos autores dieron a la empatía un componente más afectivo que cognitivo, así surgen nuevas conceptualizaciones; Stotland (1969) considera la empatía como la reacción emocional de un observador que percibe que otra persona está experimentando o va a experimentar una emoción; por su parte, Hoffman (1987) la define como una respuesta afectiva más apropiada a la situación de otra persona que a la propia; Mehrabian Epstein (1972) se refiere a la empatía como una respuesta emocional que se experimenta frente a situaciones emocionales ajenas 0 , en otras palabras, sentir lo que siente el otro (Fernández Pinto, López-Pérez y Márquez, 2008, pp.2-3).

Existen pocos estudios acerca de la validez de las prácticas adivinatorias (por ejemplo, el tarot) como un recurso psicoterapéutico (Guardia Lezcano, 2014; Semetsky, 2005; Von Stecher, 2012). El uso del término tarot es de origen italiano, surgido en el siglo XVI. Se estima que existen alrededor de 400 diferentes tipos de cartas de Tarot (Guiley, 1994). El mazo de cartas del Tarot se conforma de 78 cartas, divididas en arcanos mayores (22) y arcanos menores (56 cartas). Los arcanos mayores, representan cambios, amor, muerte, espiritualidad, aceptación, entre otras. Los arcanos menores, divididos en lo que en español llamamos "palos". Nichols (2008) sostiene que el modelo que se observa en el Tarot, no es ni más ni menos que el despliegue de la vida anímica; y, siguiendo a Jung, ve en el Tarot el despliegue de ese mundo inconsciente (para una revisión ver Alonso González, 2004). Las explicaciones externas a este acontecimiento, siguiendo a Ivtzan (2007), son primero, las que sostienen que el tarotista "lee la mente" del consultante lo que le permite realizar una devolución precisa, acertada; segundo, las que especulan con que el cliente está influenciado por la elección de las cartas o aquel que lee y expresa a través de los naipes su motivo de consulta. En el caso de aquellos que sostienen que no hay nada paranormal en la lectura del Tarot, se enfocan en procesos psicológicos haciendo énfasis en el efecto Barnum (conocido también como efecto Forer) que es la tendencia a interpretar cuestiones generales y aplicarlas a circunstancias únicas de forma precisa, al observar las reacciones del consultante, se arriesga a la lectura de eventos más exactamente -lectura en frío- como sostiene Amigo (1999). De este modo, el sujeto acepta la lectura, pero cualquier hecho relevante en sí, puede resultar peligroso porque podría ser erróneo y ocasionar algún perjuicio en el consultante (Ivtzan, 2007). La técnica Barnum necesita de buenos memoristas y de una aguda observación para "estudiar" al cliente, lo que le permite al tarotista inferir información de distintas fuentes, como, por ejemplo la forma de 
vestir, estilo, edad, posturas, forma de expresarse, etc. Se entiende que el intérprete posee conocimiento de características de personalidad y los asuntos que preocupan a los sujetos que las forman. Con dicha información preliminar, se realizaría una primera interpretación y a partir de las reacciones del cliente, las siguientes lecturas serían más exactas.

Blackmore (1983) señala que existen muchos medios por los cuales el tarotista puede obtener información para ser más preciso en la tirada y que la misma sea aceptada por todos: el intérprete puede obtener pistas por la apariencia y comportamiento del sujeto u obtener más información a través de sus respuestas o bien, la tirada de las cartas puede dar (aceptando lo paranormal) información genuina sobre el sujeto haciendo uso de conocimientos de psicología que le permitan inferir cierta información. Fueron realizados experimentos, sin embargo, fueron cuestionados por sectores escépticos ya que es muy difícil aplicar instrumentos que midan predicciones.

Ivtzan y French (2004) yuxtaponen dos perspectivas alternativas para la comprensión de los resultados de la lectura del Tarot: paranormal y no paranormal. Si el conocimiento de la lectura de los naipes es exacto, los participantes deben puntuar más alto en una lectura "real", en términos de relevancia global, en comparación con una lectura de control. Para la lectura, los participantes eligieron sus propios naipes, mientras que para el grupo control fueron dos cartas elegidas al azar. Blackmore (1983) examinó la validez de las cartas del Tarot en relación a la personalidad, sin embargo, no obtuvo resultados significativos. Sin embargo, Parra (2013, 2015) encontró que personas que indicaron tener experiencias telepáticas, premoniciones, ver el aura, sensación de presencia, y practicaban sanación mostraban mayor empatía cognitiva y emocional que quienes no tenían tales experiencias.

\section{Línea de Razonamiento}

El presente estudio enfatiza en las posibles coincidencias entre el vínculo que establece un consultante de la práctica del tarot y un psicoterapeuta con su cliente y o consultante. Debe quedar claro, naturalmente, que no se pretende extrapolar el desempeño terapéutico de un psicólogo clínico con la práctica de un tarotista, antes bien es importante mencionar que la práctica del tarot es un recurso al que un gran número de personas consulta con propósitos a menudo similares por los cuales muchos clientes de la psicoterapia también consultan (La Pietra, 2000). En consecuencia, el propósito de este estudio no pretende hacer un juicio de valor de la práctica del tarot en analogía con el desempeño de un psicoterapeuta, debido a las obvias implicaciones jurídicas, de competencia, y de entrenamiento profesional.

Tampoco se pretende un juicio de valor sobre la práctica del tarot por parte de sus practicantes, algunos (aunque no en su mayoría) serios y honestos en su saber, que reciben clientes para sus lecturas. No obstante, el foco de este estudio es el vínculo que establecen con sus clientes/consultantes. Aunque es controversial aquí determinar si un practicante de tarot establece una legítima "alianza" terapéutica, 
sin embargo, se examinará el modo en que establece este vínculo desde una perspectiva cognitiva y emocional (empatía) y si el sistema de creencias compartido entre un tarotista y su consultante empatiza o "sintoniza" del mismo modo como un psicoterapeuta establece su vínculo con el cliente mediatizado por la orientación teórica dominante del terapeuta (aunque este último aspecto no será examinado). En consecuencia, el objetivo de este estudio es evaluar la construcción de la alianza terapéutica entre el tarotista y el terapeuta con sus respectivos clientes y su relación con la empatía.

Por lo expuesto hasta aquí, lo que plantea este estudio responder es: ¿Qué elementos subyacen en la construcción de la alianza terapéutica entre terapeutas y tarotistas con sus respectivos clientes/consultantes? ¿En qué medida se relacionan el grado de empatía y el pensamiento mágico en el establecimiento de la alianza terapéutica, tanto en los psicoterapeutas como en los tarotistas con sus clientes/ consultantes?

Hipotetizamos aquí que (H1) los psicólogos y tarotistas con altas puntuaciones de empatía tenderán a reforzar la alianza terapéutica, (H2) los tarotistas puntuarán más alto en creencias (pensamiento mágico) en comparación con los psicólogos, y que (H3) los tarotistas puntuarán más alto en empatía afectiva pero bajo en empatía cognitiva, en comparación con psicólogos, quienes puntuarán más bajo en empatía afectiva y alto en empatía cognitiva.

\section{MÉTODO}

Se examinaron dos muestras. Una integrada por 37 tarotistas de ambos sexos, $10(27 \%)$ varones y $27(73 \%)$ mujeres, cuyas edades comprenden entre 23 a 62 años de edad (Media $=40,51 ; \mathrm{DT}=10,80$ ), con más de un año de dedicación (Rango= 1 a 30 años, Media= 10 años) como criterio de exclusión/inclusión (aquellos con menos de dos años de ejercicio, quedaron excluidos de la muestra), atendiendo entre 1 a 40 consultantes por mes (Media $=12$ consultantes), y entre 30 a 120 minutos por consultante (Media $=60$ minutos). La segunda muestra estuvo integrada por 42 psicólogos de ambos sexos, $6(14 \%)$ varones y $36(86 \%)$ mujeres, cuyas edades comprenden entre 24 a 61 años (Media=38,64; DT=10,76), con más de un año de dedicación (Rango $=1$ a 31 años, Media $=10$ años) como criterio de exclusión/ inclusión, atendiendo entre 2 a 200 consultantes por mes (Media $=26$ consultantes), a razón de entre 20 a 120 minutos por consultante (Media $=48$ minutos).

Se aplicó una técnica de muestreo no-probabilística intencional. Ninguno de los participantes recibió compensación económica y todos completaron los cuatro instrumentos, en forma individual. Si bien se les indicó los objetivos generales del estudio, no recibieron información respecto a las hipótesis del estudio, y se los invi-tó a participar en forma voluntaria y anónima. 


\section{Instrumentos}

Test de Empatía Cognitivo Afectiva (López-Pérez, Fernández Pinto y Abad, 2008). Es un cuestionario con 33 ítems subdivididos en 4 escalas: (1) Adopción de perspectiva, que evalúa la capacidad para la tolerancia, la comunicación y las relaciones personales; (2) Comprensión emocional, que evalúa la capacidad para reconocer los estados emocionales de los otros, así como sus intenciones e impresiones; (3) Estrés empático que se refiere a la conexión con los estados emocionales negativos de los otros y (4) Alegría empática, que hace referencia a la capacidad de compartir las emociones positivas de otras personas. El tipo de respuestas corresponden a una escala Likert, siendo $1=$ totalmente en desacuerdo, $2=$ en desacuerdo, $3=$ Neutro, $4=$ De acuerdo, y $5=$ Totalmente de acuerdo (alfa $=.79$ ).

Inventario de Alianza Terapéutica (Corbella y Botella, 2004; Corbella et al, 2011). Se basa en la formulación teórica desarrollada por Bordin a partir del constructo de Alianza Terapéutica, definiendo en la alianza tres características fundamentales: a. el terapeuta y el cliente deben estar de acuerdo en los objetivos del proceso terapéutico, b. el nivel de concordancia respecto a las tareas a realizar, c- desarrollo de un vínculo personal. El IAT tiene tres sub-escalas que evalúan, formada por reactivos de respuesta tipo Likert de 7 puntos. Hovarth y Greenberg realizaron dos versiones autoaplicables, una para ser contestada por clientes y otra para ser respondida por el terapeuta (en este estudio, se empleó el modelo que examina al terapeuta). Tracey y Kokovic (1989) plantearon la eliminación de algunos reactivos para que se facilitara su uso en el ámbito clínico, el resultado de lo cual fue el "IAT Abreviado" (WAI-S), que mantiene las 3 sub-escalas pero formado por 12 reactivos que responden a la escala Lickert de 7 puntos donde la polaridad inferior es "Nunca" y la superior es "Muy frecuentemente". Cada subescala (Vínculo, Tareas y Objetivos) está evaluada por 4 reactivos, de este modo la puntuación total (alfa $=.85)$.

Cuestionario de Creencias Paranormales (Tobacyk, 2004). Compuesto de 26 ítems diseñados para evaluar la creencia en lo paranormal. Los factores analizados fueron las convicciones religiosas tradicionales, percepción extrasensorial, brujería, superstición, espiritismo, formas de vida extraordinaria y precognición. Los 26 ítems se dividen en 5 escalas relacionadas factorialmente, derivadas de las dimensiones de las creencias paranormales. La respuesta a cada ítem tiene una escala tipo Lickert de 5 puntos. Los resultados parciales son computados en cada ítem y oscilan entre 1 y 5 puntos, donde 1 es: "En gran desacuerdo" y 5 es "Muy de acuerdo". Puntuación alta indica mayor creencia (alfa $=.90)$.

Además, se diseñó una entrevista estructurada para cada grupo con preguntas con propósitos de comparación, por ejemplo, Ejercicio de actividad (en años), Número de Clientes/Consultantes (últimos seis meses), Carga horaria por sesión/ consulta (en minutos). Luego, específicamente a los tarotistas Aprendizaje (Instructor personal, Autodidáctico, y Cursos/Seminarios), Modalidad de práctica (intuitiva, instrumental o mixta), Motivos de consulta (Conyugal, Económica, Laboral, Salud, 
Conflictos intrafamiliares, y Sentido de la Vida/Crisis espiritual), y Tipos de tarot; y para los psicólogos específicamente la Orientación teórica (Ecléctico, Cognitivo conductual, Psicoanálisis lacaniano, Psicoanálisis freudiano, y Existencialista).

\section{Análisis}

Los datos fueron cargados y procesados mediante el paquete estadístico SPSS 20. Se realizó un análisis descriptivo de los tres instrumentos empleados, para obtener frecuencias y porcentajes de las respuestas de ambos grupos, psicólogos y tarotistas. Se llevó a cabo un contraste de hipótesis sobre la normalidad de las variables estudiadas mediante un análisis de Shapiro-Wilks. Los valores obtenidos muestran que puede asumirse una distribución normalizada para las puntuaciones de los tres instrumentos. Se optó por aplicar estadística paramétrica, y evaluar el nivel de significación a una cola mediante un test $t$ de Student para comparar grupos y una $r$ de Pearson para correlacionar.

\section{RESULTADOS}

TABLA 1: RANGO, MEDIA Y DESVIO TIPICO DE EMPATIA, CREENCIAS Y ALIANZA TERAPEUTICA EN TAROTISTAS

\begin{tabular}{lrrrrrr}
\hline & \multicolumn{3}{c}{ Tarotistas } & \multicolumn{3}{c}{ Psicólogos } \\
\hline Variables & Rango & Media & \multicolumn{1}{c}{ DT } & Rango & Media & DT \\
\hline Empatía Total & $79-143$ & 110,16 & 15,32 & $77-115$ & 100,40 & 8,21 \\
Adopción de Perspectivas & $12-33$ & 25,38 & 5,16 & $15-31$ & 23,76 & 3,56 \\
Comprensión Empática & $24-40$ & 30,86 & 3,95 & $19-34$ & 27,36 & 2,76 \\
Estrés Empático & $17-36$ & 24,95 & 4,82 & $17-27$ & 21,93 & 2,63 \\
Alegría Empática & $20-36$ & 28,97 & 4,03 & $18-35$ & 27,36 & 3,7 \\
\hline F1. Empatía Cognitiva & $38-73$ & 56,24 & 8,10 & $35-63$ & 51,12 & 5,49 \\
F2. Empatía Afectiva & $39-72$ & 53,92 & 7,84 & $36-58$ & 49,29 & 4,11 \\
\hline Creencias Paranormales & $67-122$ & 95,38 & 13,60 & $30-108$ & 66,71 & 18,28 \\
\hline Alianza Positiva & $9-36$ & 24,65 & 6,51 & $19-36$ & 27,19 & 4,06 \\
Alianza Negativa & $1-10$ & 5,22 & 2,62 & $2-9$ & 4,07 & 1,62 \\
Alianza Terapéutica & $13-43$ & 29,86 & 8,02 & $23-45$ & 31,26 & 4,67 \\
\hline
\end{tabular}

En la Tabla 1 se presentan los valores de la Media y Desvío Típico de Empatía, Creencias y Alianza terapéutica en tarotistas y psicólogos. El Alfa de Cronbach de las tres escalas para ambas muestras resultó elevado, proporcionando confiabilidad a las respuestas dadas por ambos grupos (todas las alfa $<.60$ ). 
TABLA 2: ORIENTACION TEORICA DE LOS PSICOLOGOS

\begin{tabular}{lrc}
\hline Orientación teórica & $\mathrm{N}$ & $\%$ \\
\hline Ecléctico & 12 & 28,6 \\
Cognitivo conductual & 12 & 28,6 \\
Psicoanálisis lacaniano & 9 & 21,4 \\
Psicoanálisis freudiano & 7 & 16,7 \\
Existencialista & 3 & 7,1 \\
\hline
\end{tabular}

En la Tabla 2 se presentan las orientaciones teóricas dominantes entre los psicólogos. Los resultados muestran que predomina una orientación ecléctica (un abordaje no dominante de una escuela psicológica o una adaptación de varios modelos teóricos, el 28\%); el modelo cognitivo es la orientación teórica de los psicólogos encuestados y psicoanalistas, los cuales (sumados ambos) representan la primera mayoría (ambos $38 \%$ ).

TABLA 3: APRENDIZAJE, MODALIDAD DE PRÁCTICA, MOTIVOS DE CONSULTA Y TIPO DE NAIPE EN LA PRÁCTICA DEL TAROT

\begin{tabular}{lrc}
\hline Aprendizaje & $\mathrm{N}$ & $\%$ \\
Instructor personal & 23 & 62,2 \\
Autodidáctico & 21 & 56,8 \\
Cursos/Seminarios & 20 & 54,1 \\
\hline Modalidad de práctica & & \\
Sólo intuitiva & 6 & 16,2 \\
Sólo instrumental & 5 & 13,5 \\
Mixta (intuitiva y instrumental) & 33 & 89,2 \\
\hline Motivos de consulta & & \\
Conyugal & 31 & 83,8 \\
Económica & 19 & 51,4 \\
Laboral & 16 & 43,2 \\
Salud & 8 & 21,6 \\
Conflictos intrafamiliares & 13 & 35,1 \\
Sentido de la Vida / Crisis espiritual & 5 & 13,5 \\
\hline Tipos de tarot/naipes & & \\
Rider-Waite & 12 & 32,5 \\
Marsellés & 10 & 27,0 \\
Otros & 6 & 16,2 \\
Egipcio & 4 & 10,8 \\
Ángeles & 3 & 8,1 \\
Legacy & 2 & 5,4 \\
\hline
\end{tabular}

En la Tabla 3 se presentan la instrucción recibida por el tarotista (Aprendizaje), la modalidad de práctica del tarot, y el motivo de consulta más frecuente. La instrucción personalizada es predominante (62\%), seguido de aprendizaje sin instructor (autodidáctica, 56\%), y a través de cursos y seminarios (54\%). La modalidad de práctica más frecuente es una forma "mixta" (89\%) entre la intuitiva 
(que por sí sólo fue de 16\%) o la instrumental (por si sola fue de 13\%). Los tipos de naipes más utilizados por los tarotistas son los Rider-Waite (32,5\%) Marsellés (27 \%) y Egipcio (10,8 \%).

TABLA 4: COMPARACION DE EJERCICIO DE ACTIVIDAD, NÚMERO DE CLIENTES/ CONSULTANTES Y CARGA HORARIA POR SESION ENTRE TAROTISTAS Y PSICOLOGOS

\begin{tabular}{lrrrrrr}
\hline & \multicolumn{2}{c}{$\begin{array}{c}\text { Tarotistas } \\
(n=37)\end{array}$} & \multicolumn{2}{c}{$\begin{array}{c}\text { Psicólogos } \\
(n=42)\end{array}$} & & \\
\hline & Media & \multicolumn{1}{c}{ DT } & Media & DT & t & $\mathrm{p}$ \\
\cline { 2 - 8 } Antigüedad (en años) & 10,49 & 7,03 & 10,38 & 9,34 & 0,05 & n.s. \\
\hline Clientes/Consultantes $^{*}$ & 11,95 & 10,03 & 26,21 & 38,60 & 2,18 &, 032 \\
\hline Carga horaria** $^{*}$ & 60,54 & 19,81 & 48,69 & 9,69 & 3,43 &, 026 \\
\hline
\end{tabular}

* Ultimos seis meses.

** Por sesión/consulta, en minutos.

Se llevó a cabo una comparación en la antigüedad en el ejercicio de actividad, el número promedio de clientes/consultantes, y la carga horaria por sesión (en minutos) entre tarotistas y psicólogos mediante la $t$ de Student. No se encontraron diferencias entre ambos grupos en antigüedad, pero se observó que los psicólogos tienden a tener -en promedio- más clientes que los consultantes en la práctica del tarot $(\mathrm{p} .=.032)$, pero la carga horaria para la atención de la consulta/sesión es levemente superior para los tarotistas en comparación con los psicólogos $(\mathrm{p} .=.026)$ (ver Tabla 4).

TABLA 5: COMPARACION EN EMPATIA, CREENCIAS Y ALIANZA TERAPEUTICA ENTRE TAROTISTAS Y PSICOLOGOS

\begin{tabular}{lrrrrrr}
\hline & \multicolumn{2}{c}{$\begin{array}{c}\text { Tarotistas } \\
(n=37)\end{array}$} & \multicolumn{2}{c}{$\begin{array}{c}\text { Psicólogos } \\
(n=42)\end{array}$} & & \\
\hline & Media & \multicolumn{1}{c}{ DT } & Media & \multicolumn{1}{c}{ DT } & \multicolumn{1}{c}{$\mathrm{t}$} & \multicolumn{1}{c}{$\mathrm{p}$} \\
\hline Empatía (Total) & 110,16 & 15,32 & 100,40 & 8,21 & 3,58 &, 001 \\
1. Perspectiva & 25,38 & 5,16 & 23,76 & 3,56 & 1,64 & n.s. \\
2. Comprensión Empática & 30,86 & 3,95 & 27,36 & 2,76 & 4,60 & $<, 001$ \\
3. Estrés Empático & 24,95 & 4,82 & 21,93 & 2,63 & 3,50 & $<, 001$ \\
4. Alegría Empática & 28,97 & 4,03 & 27,36 & 3,75 & 1,84 & n.s. \\
F1. E. Cognitiva & 56,24 & 8,10 & 51,12 & 5,49 & 3,32 &, 001 \\
F2. E. Afectiva & 53,92 & 7,84 & 49,29 & 4,11 & 3,34 &, 001 \\
\hline Creencias & 95,38 & 13,60 & 66,71 & 18,28 & 7,81 & $<, 001$ \\
\hline Alianza Terapéutica & 29,86 & 8,02 & 31,26 & 4,67 & $-0,96$ & n.s. \\
Vínculo & 8,13 & 2,65 & 7,29 & 1,63 & 1,70 & n.s. \\
Tareas & 11,51 & 3,12 & 11,79 & 2,06 & $-0,46$ & n.s. \\
Objetivos & 7,22 & 2,47 & 9,14 & 1,45 & $-4,27$ & $<.001$ \\
$\quad$ F1. Alianza Positiva & 24,65 & 6,51 & 27,19 & 4,06 & $-2,10$ &, 038 \\
F2. Alianza Negativa & 5,22 & 2,62 & 4,07 & 1,62 & 2,38 &, 021 \\
\hline
\end{tabular}


Se llevó a cabo una comparación en empatía, creencias y alianza terapéutica entre tarotistas y psicólogos mediante la $t$ de Student. Se encontraron diferencias en Empatía (p. <.001), en particular Comprensión Empática (p. < .001) y Estrés Empático (p. $<.001)$, así como en las dimensiones Cognitiva y Afectiva de la Empatía (ambas p. $=.001$ ), donde los tarotistas puntuaron significativamente más alto que los psicólogos. Además, los tarotistas mostraron mayor nivel de creencia que los psicólogos ( $\mathrm{p} .<.001)$. Aunque no se encontraron diferencias en Alianza Terapéutica entre ambos grupos, sin embargo, se encontró mayor alianza positiva en los psicólogos en comparación con los tarotistas $(\mathrm{P} .=.038)$, pero en sentido inverso, mayor alianza negativa en tarotistas en comparación con los psicólogos ( $\mathrm{p}$. $=.021$ ). Los psicólogos también puntuaron alto en Alianza focalizada en "objetivos" entre cliente/consultante en comparación con los tarotistas (p. <.001) (ver Tabla 5).

TABLA 6: CORRELACION ENTRE EMPATIA COGNITIVA/AFECTIVA Y TOTAL Y ALIANZA TERAPEUTICA EN TAROTISTAS Y PSICOLOGOS

\begin{tabular}{lcccccc}
\hline & \multicolumn{3}{c}{$\begin{array}{c}\text { Tarotistas } \\
(n=37)\end{array}$} & \multicolumn{3}{c}{$\begin{array}{c}\text { Psicólogos } \\
(n=42)\end{array}$} \\
\hline & $\begin{array}{c}\text { Empatía } \\
(\text { Total) }\end{array}$ & $\begin{array}{c}\text { Empatía } \\
\text { Cognitiva }\end{array}$ & $\begin{array}{c}\text { F2. } \\
\text { Empatía } \\
\text { Afectiva }\end{array}$ & $\begin{array}{c}\text { Empatía } \\
\text { (Total) }\end{array}$ & $\begin{array}{c}\text { F1. } \\
\text { Empatía } \\
\text { Cognitiva }\end{array}$ & $\begin{array}{c}\text { F2. } \\
\text { Empatía }\end{array}$ \\
\hline Creenctiva \\
\hline Alianza Positiva &, $732^{* *}$ &, $676^{* * *}$ &, $733^{* * *}$ &, $333^{* *}$ &, $350^{*}$ &, 198 \\
Alianza Negativa &, $622^{* *}$ &, $608^{* * *}$ &, $587^{* * *}$ &, 199 &, 239 &, 078 \\
Alianza &, $506^{* *}$ &, $481^{* * *}$ &, $493^{* * *}$ &,- 013 &, 114 &,- 178 \\
\hline
\end{tabular}

${ }^{*} p<.05 ;{ }^{* *} p<.01 ;{ }^{* *} p<.001$

Se llevó a cabo una correlación mediante el coeficiente $r$ de Pearson entre empatía cognitiva/afectiva (y total) y alianza terapéutica en ambos grupos, tarotistas y psicólogos. Respecto a Creencias, si bien se encontraron correlaciones significativas entre empatía y creencias paranormales, las correlaciones más robustas se presentaron en el grupo de tarotistas en comparación con los psicólogos $(r=73 \mathrm{y} r=$ .33 , respectivamente); los tarotistas puntuaron más alto en empatía afectiva $(r=.73)$ en comparación con los psicólogos $(r=.19)$, lo mismo para empatía cognitiva, donde los tarotistas obtuvieron los valores más altos $(r=.67 \mathrm{y} r=.35$, respectivamente). Era esperable hallar valores significativos en creencias en los tarotistas y no en psicólogos, y probablemente la correlación con empatía en el primer grupo esté ligada al pensamiento mágico de los consultantes. Es así que encontramos que la relación entre creencias y empatía afectiva no fue significativa para los psicólogos. Respecto a Alianza, se encontraron correlaciones muy significativas entre Alianza y Empatía en los tarotistas ( $\mathrm{p} .<.001)$, pero ninguna relación en los psicólogos $(r=$ .16) (ver Tabla 6). 
TABLA 7: CORRELACION ENTRE ALIANZA TERAPEUTICA Y CREENCIAS PARANORMALES EN TAROTISTAS Y PSICOLOGOS

\begin{tabular}{lccc}
\hline & $\begin{array}{c}\text { Alianza } \\
\text { Positiva }\end{array}$ & $\begin{array}{c}\text { Alianza } \\
\text { Negativa }\end{array}$ & $\begin{array}{c}\text { Alianza } \\
\text { Terapéutica }\end{array}$ \\
\hline Creencias Tarotistas &, $564^{* * *}$ &, $522^{* * *}$ &, $628^{* * *}$ \\
Creencias Psicólogos &, 202 &, 034 &, 188 \\
\hline
\end{tabular}

${ }^{*} p<.05 ;{ }^{* *} p<.01 ;{ }^{* * *} p<.001$

Se llevó a cabo una correlación mediante el coeficiente $r$ de Pearson entre alianza terapéutica y creencias paranormales en ambos grupos, tarotistas y psicólogos. Respecto a Creencias, se encontraron correlaciones significativas entre alianza terapéutica y creencias paranormales en el grupo de tarotistas (p. $<.001)$, pero no se encontró ninguna correlación en el grupo de psicólogos (ver Tabla 7).

\section{CONCLUSIONES}

El objetivo general de este estudio fue evaluar la construcción de la alianza terapéutica entre el tarotista y el psicólogo con sus clientes/clientes y su relación con la empatía y el sistema de creencias del tarotista y el psicólogo, más específicamente, comparar la alianza terapéutica, el grado de empatía, y la medida de pensamiento mágico entre psicólogos y tarotistas, y relacionar la empatía, la alianza terapéutica y el pensamiento mágico en psicólogos y tarotistas.

La hipótesis central que sugiere que psicólogos y tarotistas con alta empatía tenderán a tener mayor alianza terapéutica con sus clientes fue parcialmente confirmada para los tarotistas, pero no para los psicólogos (fue significativa la correlación entre Alianza terapéutica y Empatía en tarotistas, p. $<, 001$ mientras que no hubo ninguna relación para el grupo de psicólogos). Teniendo en cuenta que el concepto de "empatía" es importante para la psicología, los resultados obtenidos para el grupo de psicólogos no eran lo esperado. Entendiendo básicamente que la empatía se define como la capacidad de ponerse en lugar del otro, se planteó la hipótesis central en base a este constructo y al de Alianza Terapéutica del cual aquél formaría parte. Sin embargo, los tarotistas, como mencionáramos más arriba, puntuaron significativamente más alto que los psicólogos. Andrade González (2005), se refiere a la Alianza Terapéutica como una compleja red de nexos entre el cliente y el terapeuta entre las que se encuentran la empatía, la confianza mutua y la aceptación, por ello es destacable el resultado obtenido en la correlación entre ambos constructos en el grupo de psicólogos para quienes no habría relación entre la empatía y su influencia en la formación de la Alianza terapéutica.

La H3 que sugiere que los tarotistas puntuarían más alto en empatía afectiva, pero bajo en empatía cognitiva en comparación con los psicólogos quienes puntuarán más bajo en empatía afectiva y alto en empatía cognitiva, no fue confirmada en estos términos, aunque ciertamente los tarotistas mostraron mayor capacidad empática que los psicólogos, mayor comprensión empática y estrés 
empático, en otras palabras, los tarotistas mostraron ser más empáticos en relación con la habilidad de ponerse en el lugar de su consultante, y experimentar sentimientos con sus problemáticas de consulta así como eventos positivos de la vida de sus clientes.

Sin embargo, hay otros análisis reveladores en este estudio que merecen ser examinados respecto al sistema de Creencias de los tarotistas y los psicólogos, y en la construcción de la Alianza Terapéutica. Por ejemplo, aunque no se encontraron diferencias en la construcción de la alianza terapéutica (un atributo instrumental del rol de la psicoterapia) entre psicólogos y tarotistas, sin embargo, los psicólogos mostraron indicadores más positivos ("Estamos de acuerdo en aquellos temas o situaciones que son importantes procesar") que los tarotistas, y éstos indicadores más negativos ("Mi consultante/cliente y yo tenemos diferencias en cuanto a cuáles son sus problemas más importantes") que los psicólogos.

Otra diferencia significativa se presenta en el sistema de creencias de ambos grupos, siendo los tarotistas quienes sostienen creencias asociadas a sus prácticas y al contexto de las mismas a y la demanda de sus consultantes. Esto estaría confirmando la $\mathrm{H} 2$ que sostiene que los tarotistas puntuarían más alto en pensamiento mágico (creencias) en comparación con los psicólogos. Otro dato relevante surge de la interacción entre pensamiento mágico (creencias) y alianza terapéutica en relación con la empatía, por ejemplo, los tarotistas mostraron mayor empatía afectiva y cognitiva en relación con las creencias, lo cual podría sugerir que el éxito en el vínculo que establece con su consultante está asociado a compartir un sistema de creencias en la eficacia de la práctica del tarot como saber esotérico. Posiblemente, esta relación entre el vínculo empático con el cliente y el sistema de creencias refuerza la alianza que establece con su consultante, aun cuando el propósito de la práctica del tarot guarda diferencias con la psicoterapia (recordemos, como se expresó en este mismo trabajo, que Cernadas (2006) señala la importancia de reflexionar sobre el sistema de creencias del sujeto para poder comprender su comportamiento). No obstante, el rol que cumple el tarotista podría servir de igual modo (a la terapia).

Es notable, que esta misma relación no está presente en los psicólogos, en particular la empatía con el cliente como un refuerzo de la alianza terapéutica. Podría esperarse una ausencia de la relación entre pensamiento mágico y la empatía -aunque sería deseable en aquellos terapeutas en condiciones de "sintonizar" con el sistema de creencias de sus clientes como un aliado de la psicoterapia (por ejemplo, aquellos clientes con creencias religiosas o espirituales en el contexto de la psicoterapia), pero es cuanto mucho alarmante una falta de relación entre la construcción de la alianza terapéutica y la empatía, considerando que el éxito de la alianza terapéutica -y por extensión de la eficacia de un sistema terapéuticodescansa en la empatía cognitiva y afectiva como condición necesaria para la psicoterapia. Este mismo efecto se presenta en la relación entre el pensamiento mágico y la alianza terapéutica como un signo que "cristaliza" de algún modo el 
vínculo entre el consultante y el tarotista, tal como indican los resultados obtenidos en este estudio a diferencia de la ausencia de tal relación entre los psicólogos. En síntesis, estos resultados sugieren que las emociones y las creencias de los clientes/ consultantes -no solo las creencias esotéricas; en un sentido amplio, sus esquemas cognitivos como un todo integrado- no deberían ser un obstáculo sino un "atajo" para empatizar o crear un puente funcional para una psicoterapia exitosa.

Los tarotistas, aunque por definición no operan como terapeutas, sin embargo, cumplen un rol en cierta medida semejante en el modo en que establecen el vínculo con su consultante a causa de que sus principales temas de consulta son los problemas conyugales, económicos, y conflictos intrafamiliares que, en cierto grado, no difieren de las demandas que llegan a los psicólogos; además dedican más tiempo a sus clientes, si bien los psicólogos atienden un número superior de clientes que los consultantes de las prácticas de tarot.

Naturalmente, debemos considerar las diferencias entre la práctica del tarot y la psicoterapia, usualmente el tarotista no implementa un dispositivo terapéutico per se como lo hace un psicólogo; tampoco establece un abordaje específico a lo largo de un periodo del mismo modo que un psicólogo o un psicoanalista, y el compromiso emocional y cognitivo podría ser claramente diferencial. Pero es evidente, que los roles parecen ser semejantes y que el tarotista (o al menos el contexto de su práctica) favorece o vehiculiza mejor, de algún modo, la alianza con su consultante, o al menos eso subjetivamente es lo que experimenta el tarotista probablemente a causa de su facilidad por "entrar en sintonía" con el universo simbólico y el sistema de creencias (pensamiento mágico) que es funcional para el consultante, reforzando y modulando el vínculo empático tanto a nivel emocional como cognitivo. De este modo, produce una alianza terapéutica experienciada subjetivamente por el tarotista con sus clientes, que posiblemente sea más flexible que el establecido por el terapeuta.

El psicólogo podría estar más comprometido e involucrado con un modelo (o varios) teórico o estar convencido y confiar en la eficacia de sus estrategias y/o técnicas terapéuticas, a diferencia del tarotista, quien posiblemente no se encuentre tan comprometido con un modelo teórico específico, con mayor flexibilidad en su sistema de creencias y capacidad de adaptarse más fácilmente a los cambios cognitivos y afectivos de sus consultantes; por ejemplo, la interpretación de un naipe del tarot podría tener muchas potenciales interpretaciones, aun contradictorias entre sí, pero el tarotista adapta su intervención más "dirigido-a-las-necesidades" de su consultante más que "dirigido-a-la-eficacia" del recurso terapéutico, sin entrar en conflicto con éste. En cambio, en la psicoterapia, el compromiso de cambio que exige está mediatizado por la alianza terapéutica (por definición, un recurso "patrimonial" de la psicoterapia) el cual conlleva mayores ajustes cognitivos, más tiempo de proceso, asunción de responsabilidades, y auto-aceptación de los cuales el terapeuta es más responsable (e incluso el motor) que lo que un consultante recibiría típicamente de su tarotista. 
Quizás en consonancia con los resultados obtenidos y analizados hasta aquí, se encontraron limitaciones al momento de administrar los instrumentos necesarios para la investigación. Al comenzar a buscar la muestra para la administración de las encuestas, los tarotistas se prestaron a responder con cordialidad y totalmente despojados de prejuicios, incluso solicitaron que al finalizar la investigación se les comunicaran los resultados. Sin embargo, conseguir la muestra de psicólogos fue mucho más compleja, ya que cuando se los invitaba a participar de la investigación (aclarándoles que las encuestas eran anónimas y con fines estadísticos), muchos se negaban directamente, otros solicitaban completarlas y luego entregarlas (se las llevaron y nunca las devolvieron), algunos respondieron que no era ético someter a los psicólogos a semejantes encuestas, otros insistieron con el secreto profesional y unos pocos las respondieron aclarando que se sentían "analizados", sin embargo, un número importante de profesionales accedieron gustosos a responder, incluso a ser intermediarios con otros colegas e interesados en los resultados de la investigación. Sería interesante como tema de una próxima investigación indagar acerca de las resistencias de los psicólogos para participar de la investigación (como se hizo referencia en las Conclusiones del presente trabajo) como así también los motivos por los cuales fueron tan significativas las diferencias halladas en la formación de la alianza terapéutica y la empatía, en la presente investigación.

\section{Notas}

* Alejandro Parra es Doctor en psicología (UCES) y psicoterapeuta en la práctica privada. Es profesor graduado en Psicología por la Universidad Abierta Interamericana de Buenos Aires, y docente adjunto e investigador universitario (Categoría IIA. Res. CSN.4140/13). Es director de la Diplomatura Superior en Psicologia Transpersonal en la Universidad Abierta Interamericana. Su email es: rapp@fibertel.com.ar

** Claudia Sciacca es psicóloga graduada por la Universidad Abierta Interamericana. Es Profesora en Historia (1996) Instituto Superior de Profesorado Pbro. Dr. A.M. Saenz, Profesora para la Enseñanza Primaria (1991) Instituto Ntra. Sra. Del Sagrado Corazón, con un post-grado en Informática Educativa (1998). Actualmente es Profesora en Comunicación, Cultura y Educación I. Ntra. Sra. del Sagrado Corazón, (Fcio. Varela), y Directora del Nivel Secundario, Comunidad Educativa Modelo 2000 de la Ciudad de Florencio Varela, Argentina. Su email es: csciacca@intervar.com.ar

\section{AGRADECIMIENTOS}

A los profesionales, psicólogos y tarotistas, quienes voluntariamente aceptaron participar de este estudio, sin cuyos valiosos aportes el presente trabajo no hubiese sido posible. El presente estudio es una versión abreviada de la tesis de pregrado de Claudia Sciacca para la Facultad de Psicología \& Relaciones Humanas de la Universidad Abierta Interamericana, en cooperación con el Instituto de Psicología Paranormal, ambos en Buenos Aires, Argentina. 


\section{Referencias bibliográficas}

Alonso González, J. (2004). La psicología analítica de Jung y sus aportes a la psicoterapia. Universitas Psychologica, 3(1), 55-70.

Amigo, I. (1999). Astrología. El mito de las estrellas. Psicothema, 11(2), 447-448.

Andrade González, N. (2005). La alianza terapéutica. Clínica y Salud, 16(1), 9-29.

Baringoltz, S. (2005, agosto). Aportes cognitivos a la visión del terapeuta y vínculo terapéutico. Ponencia presentada en el IV Congreso Mundial de Psicoterapias, Buenos Aires, Argentina.

Blackmore, S. (1983). Divination with Tarot cards: An empirical study. Journal of the Society for Psychical Research, 52, 97-99.

Botella, L. y Feixas, G. (1998). Teoría de los constructos personales: Aplicaciones a la práctica psicológica. Barcelona: Laertes.

Bowlby, J. (1995). Una base segura. Barcelona: Paidós.

Cernadas, C. (2006). El poder de los símbolos. Magia, enfermedad y acto médica. Revista del Hospital Italiano de Buenos Aires, 26(3), 87-93.

Corbella, S., y Botella, L. (2003). La alianza terapéutica: historia, investigación y evaluación Anales de Psicología, 19(2), 205-221.

Corbella, S., Botella, L.(2004. Psychometric properties of the Spanish version of the Working Alliance Theory of Change Inventory (WATOCI). Psicothema, 16, 23-43.

Corbella, S., Botella, L., Gómez, A., Herrero, y O., Pacheco, M. (2011). Características psicométricas de la versión española del Working Alliance Inventory-Short (WAI-S). Anales de Psicología, 27(2), 298-301.

Etchevers, M., González, M., Simkin, H. (2012). Principales desarrollos y enfoques sobre alianza y relación terapéutica. Anuario de investigaciones, $X X$. Facultad de Psicología, Universidad de Buenos Aires.

Feeney, J. y Noller, P. (2001). Apego adulto. Bilbao: Desclèe de Brouver.

Fernández-Pinto, I., López Pérez, B., y Márquez, M. (2008). Empatía: Medidas, teorías y aplicaciones en revisión. Anales de Psicología, 24(2), 284-298.

Guardia Lezcano, J. (2014). Tarot babilónico en relación a la psicología y como primeras formas de subjetividad. VI Congreso Internacional de Investigación y Práctica Profesional en Psicología. Buenos Aires: Facultad de Psicología, Universidad de Buenos Aires.

Guiley, Rosemary Ellen, (1994). Enciclopedia de lo Mistico y lo Paranormal. Miami, FL: Harper Collins Publishers.

Hoffman, M. (1987). The contribution of empathy to justice and moral judgment. En J. Eisenberg y J. Strayer (Eds.), Empathy and its development (pp. 47-80). New York, NY: Cambridge University Press.

Ivtzan, I. (2007). Tarot cards: A literatura review and evaluation of psychic versus psychological explanations. Journal of Parapsychology, 71, 139- 149.

Ivtzan, I. y French, C. (2004). Testing the validity of Tarot Cards: can we distinguish between a real and a control reading? Parapsychological Association Convention, 437-439.

La Pietra, D. (2000). El tarot en la ciudad de Buenos Aires. La búsqueda de sentido a través de las prácticas mágicas. [Recuperado el 28.5.13 de http://redbus.usal.edu.ar/cgi-bin/koha/opacdetail.pl?biblionumber $=44754]$

Lossa, L., Corbella, S., \& Botella, L. (2012). Estilo de apego del paciente en la construcción de la alianza terapéutica. Boletín de Psicología, 105, 91-103.

Luborski, L. (1976). Helping Alliance in psychotherapy. En J.L. Claghorn (Ed.), Successful psychotherapy. New York, NY. Bruner/Mazel.

Mehrabian, A. Epstein, N. (1972). A measure of emotional empathy. Journal of Personality, 40, 525-543.

Nichols, S. (1988 / 2008). Jung y el Tarot. Un viaje arquetípico. Barcelona: Kairós.

Ojeda, M. (2010). Origen y evolución de la alianza terapéutica. Revista GPU, 6, 284-295.

Olivera, J., Braun, M., Roussos, A. (2011). Instrumentos para la evaluación de la empatía en psicoterapia. Revista Argentina de Clínica Psicológica, 20, 121-132.

Parra. A. (2013). Cognitive and emotional empathy in relation to five paranormal/anomalous experiences. North American Journal of Psychology, 15(3), 405-412.

Parra. A. (2015). Personality traits associated with premonition experience: Neuroticism, extraversion, empathy, and schizotypy Journal of the Society for Psychical Research, 79(1), 1-10.

Piedrabuena, S. (2007). Cualidades personales del terapeuta en opinión de los pacientes y en relación a la percepción de mejoría. Tesis de maestría no publicada, Universidad de Belgrano, Ciudad Autónoma de Buenos Aires, Argentina. 
Semetsky, I. (2005). Integrating Tarot readings into counselling and psychotherapy. Spirituality and Health International, 6, 81-94.

Stotland, E. (1969). Exploratory investigations of empathy. En Berkowitz(Ed.), Advances in Experimental Social Psychology, 4. San Diego, CA: Academic Press.

Tobacyk, J.J. y Mitchell, T.E. (1987). Out-of-body experience status as a moderator of effect of narcicism on paranormal belief. Psychological Reports 60, 440-442.

Von Stecher, P. (2012). Una lectura semiótica-discursiva del Tarot y el estudio de un caso. AdVersus, 9, 151-165. 